\title{
A Nomogram Based on Preoperative Clinical Bio-Indicators to Predict 5-year Survivals for Patients with Gastric Cancer After Radical Gastrectomy
}

This article was published in the following Dove Press journal:

Cancer Management and Research

\author{
Qi Hang Yin ${ }^{1,2}$ \\ Bin Zheng Liu ${ }^{3}$ \\ Meng Qing $X u^{1,4}$ \\ Lin Tao ${ }^{1,2}$ \\ Kui Wang ${ }^{5}$ \\ Feng $\mathrm{Li}^{1,6}$ \\ Wen Jie Zhang ${ }^{1,2}$
}

'Department of Pathology, The First Affiliated University Hospital, Shihezi University School of Medicine, Shihezi, Xinjiang, People's Republic of China; ${ }^{2}$ The Key Laboratories for Xinjiang Endemic and Ethnic Diseases, Shihezi University School of Medicine, Shihezi, Xinjiang, People's Republic of China; ${ }^{3}$ Department of Pathology, The First People's Hospital, Jiande, Zhejiang, People's Republic of China; ${ }^{4}$ Department of Gastroenterology and Hepatology, Suzhou City Hospital, Suzhou, Anhui, People's Republic of China; ${ }^{5}$ Department of Preventive Medicine, Shihezi University School of Medicine, Shihezi, Xinjiang, People's Republic of China; 'Department of Pathology, Beijing Chaoyang Hospital, Capital Medical University, Beijing, People's Republic of China
Correspondence: Wen Jie Zhang Department of Pathology, Shihezi University School of Medicine, 59 North 2nd Road, Shihezi, Xinjiang 832002,

People's Republic of China

Tel +86- I39-8627-7576

Fax +86-993-205-7I36

Email 1959045791@qq.com
Purpose: This study aimed to improve the prediction of postoperative survival outcomes for patients with gastric cancer (GC) using a nomogram based on preoperative bio-indicators.

Patients and Methods: This retrospective study included $303 \mathrm{GC}$ patients who had undergone radical gastrectomy from 2004 to 2013 at the First Affiliated Hospital, Shihezi University. The patients were followed up for 175 months after surgery and then divided into short-term $(\mathrm{n}=201)$ or long-term $(\mathrm{n}=102)$ survival groups. We used an expectationmaximization method to fill any missing data from the reviewed patient files. We then employed the Cox proportional hazard regression to identify biochemical markers that could predict 5-year overall survival (OS) as an endpoint among GC patients. Based on the results from the biochemical analysis, we developed a nomogram and assessed its performance and reliability.

Results: The variables significantly associated with OS in a multivariate analysis were age, body mass index (BMI), cell differentiation, high-density lipoprotein cholesterol (HDL-C), as well as serum potassium or serum magnesium. Combining all these predictors allowed us to establish a nomogram $(\mathrm{C}$-index $=0.701)$ whose accuracy of predicting survival was higher than the TNM staging system established by the 8th American Joint Committee on Cancer ( $\mathrm{C}$-index $=0.666 ; \mathrm{p}=0.016$ ). Furthermore, decision curve of this nomogram was shown to have an ideal net clinical benefit rate.

Conclusion: We have developed an algorithm using preoperative bio-indicators and clinical features to predict prognosis for GC patients. This tool may help clinicians to strategize appropriate treatment options for GC patients prior to surgery.

Keywords: gastric cancer, 5-year overall survival, prognosis, nomogram

\section{Introduction}

Gastric cancer (GC) poses an imminent threat to public health in the world, particularly in China. According to the 2018 Global Cancer Statistics, the estimated 456,124 new GC cases and 390,182 new deaths due to GC were reported in China, representing $44.1 \%$ and $49.9 \%$ of world new GC cases and GC-related deaths, respectively. Besides, out of all malignant tumors, China ranks second in the GC morbidity and mortality in the world. ${ }^{1,2}$

In recent years, advanced screening methods coupled with improved public health awareness have put the GC morbidity and mortality under control. ${ }^{3}$ 
However, the prognosis of advanced gastric cancer (AGC) remains poor. ${ }^{2}$ Radical gastrectomy remains a mainstay treatment for AGC. Given that TNM clinical staging is not available before gastrectomy, ${ }^{4}$ choosing appropriate surgical strategies usually depends on patient-specific clinical information and patients' general health ${ }^{5}$ making it difficult for clinicians to employ effective surgical options which affect prognosis for GC patients. ${ }^{6}$ Therefore, a method that could predict patients' prognosis prior to surgery would be very helpful for physicians to determine surgical options as well as postoperative treatment decisions.

A number of scoring and risk-stratification systems that utilize various demographic information and postoperative clinical parameters have been suggested to be able to predict prognosis for GC patients. ${ }^{7-10}$ However, there is little research using preoperative clinical information to predict the long-term survival outcomes after radical GC gastrectomy. In this study, we have first investigated the feasibility of using preoperative bio-indicators to predict GC prognosis. Here we report a 5 -year survival algorithm by establishing and validating a nomogram employing preoperative clinical bio-indicators.

\section{Patients and Methods}

\section{Ethics Statement}

This study was approved by the Institutional Ethics Review Board (IERB No. SHZ2010LL03) at the First Affiliated Hospital, Shihezi University School of Medicine. The IERB had waived the requirement for written informed consent due to anonymous analyses of the data. The standard university hospital guidelines were followed, which are in line with the Declaration of Helsinki outlining the ethical principles for medical research that involve human samples.

\section{Patients and Follow-up}

We reviewed medical records of 500 patients who underwent radical gastrectomy for GC between January 1, 2004 and June 30, 2013 at the First Affiliated Hospital of the School of Medicine, Shihezi University. Two senior pathologists re-evaluated the GC diagnoses and identified those who had: gastric adenocarcinoma or squamous carcinoma during postoperative pathology, undergone blood chemistry tests before surgery and available records of body weight and height without chemo-radiotherapy before surgery. A total of 180 patients who had preoperative chemoradiotherapy or other treatment that affected blood biochemical levels $(\mathrm{n}=126)$; gastric stump cancer $(n=31)$; combined with other malignant tumors $(\mathrm{n}=15)$; or incomplete clinicopathological information $(n=8)$ were excluded from the study. Ultimately, 320 patients (237 men and 83 women, mean age 61.7 [range, 27-86] years) were included in the study and were followed up for 175 months (14.6 years) after surgery. The median follow-up time was 104 months. It should be noted that the patients studied here were from a city, Shihezi City in China's far west, where a vast majority of residents were migrants from all over the country, ${ }^{11}$ suggesting the heterogeneity of this patient population.

\section{Clinical and Biochemical Features}

In the present study, we only included patients who had not received chemo-radiotherapy before surgery, which allowed us to evaluate the effectiveness of the nomogram in predicting 5-year survival for GC patients after radical gastrectomy. We obtained clinical data including age, sex, height, weight, phone number, address, medical history and clinicopathological information from the patients' medical records. Using the collected clinical data, we calculated the body mass index $\left(\mathrm{BMI} ; \mathrm{kg} / \mathrm{m}^{2}\right)$. Patients were grouped according to the standards set by the Chinese Working Group on Obesity. ${ }^{12}$

Blood chemistry tests were performed in the Clinical Biochemistry Laboratories at the First Affiliated Hospital, Shihezi University School of Medicine. Normal reference intervals for the blood biochemical indicators were in accordance with the Health Industry Standards of the People's Republic of China. ${ }^{13-16}$

The TNM staging and the degree of cell differentiation were based on post-resection histopathology. The staging was determined based on surgical findings as described by the 8th American Joint Committee on Cancer (AJCC) TNM staging system. ${ }^{4}$

\section{Statistical Analyses}

Descriptive data were presented as a mean $\pm \mathrm{SD}$, while categorical data were presented as a percentage. Comparison between the different groups was analyzed using the Student's $t$-test or chi-square test/Fisher's exact test for continuous or categorical variables, respectively. Survival curves were drawn by the Kaplan-Meier method. On the other hand, to identify independent variables, factors that were significant in univariate or multivariable analysis were included in Cox proportional hazard 
regression analysis. The percentages of missing data in patient files were $12.7 \%$. And missing mechanism was missing at random. Expectation-maximization was a convenient, effective and reasonable imputation method in dealing with missing data. ${ }^{17}$ Expectation-maximization calculates maximum likelihood estimation or posterior probability distribution through iteration. We used an expectation-maximization method to fill any missing data from the reviewed patient files.

All statistical analyses were carried out using Statistical Product and Service Solutions (SPSS) 22.0 software (SPSS Inc, Chicago, IL, USA), R (version 3.4.3; http://www.R-project.org) and EmpowerStats software (http://www.empowerstats.com). Nomogram discrimination power was evaluated by concordance index (C-index), which is equivalent to the area under the receiver operating characteristic curve. Moreover, for comparison, we calculated the C-index of the 8th AJCC TNM staging system. The area under the curve (AUC) ranges between 0 and 1 , where 1 shows perfect concordance, while 0.5 indicates no better concordance than chance. We constructed calibration plots and validated them with 500 bootstrap repetitions to reduce bias. Furthermore, we employed the decision curve analysis (DCA) to evaluate the clinical application value of the nomogram. Whereas a p-value of less than 0.05 is usually considered statistically significant, a p-value of less than 0.15 was considered statistically significant in our Cox proportional hazard regression univariate analysis.

\section{Results}

\section{Demographic and Biochemical Data}

Of the 500 patients reviewed, 320 patients were eligible for inclusion. However, 17 (5.3\%) patients were excluded from the analysis because of a lack of or inconsistent information regarding the biochemical results or followup periods. The study reviewed and analyzed data from the remaining 303 patients (Figure S1). By October 2, 2018, $208(68.6 \%)$ patients had died. Overall, 201 patients died within 5 years (short-term survival group) while 102 patients survived longer than 5 years (long-term survival group). The detailed characteristics of the study population are as summarized in Table 1.

Patients in the long-term survival group were significantly younger compared to those in the short-term survival group $(59.1 \pm 11.8$ years vs $63.0 \pm 11.1$ years, $p=0.025)$.
Both the BMI and cell differentiation were better in the long-term survival group $(\mathrm{p}=0.005)$. In addition, shortterm survival group had more advanced cancer stages compared to the long-term survival group. This phenomenon was true in tumor invasion (T), lymph node metastasis (N), distant metastasis (M) and TNM stage $(\mathrm{p}<0.001)$.

There were significant differences in albumin (Alb) levels, the albumin/globulin (A/G) ratio, blood glucose (Glu) levels, total cholesterol (TC), high-density lipoprotein cholesterol (HDL-C), low-density lipoprotein cholesterol (LDL-C) or serum sodium between the long-term and short-term survival groups $(\mathrm{p}=0.003, \mathrm{p}=0.012, \mathrm{p}=0.014$, $\mathrm{p}=0.043, \mathrm{p}<0.001, \mathrm{p}=0.011, \mathrm{p}=0.023$, respectively).

\section{Prognosis Factors Associated with the Overall Survival in Gastric Cancer}

The mean follow-up for the 303 patients was $109.1 \pm 3.1$ months, while the median follow-up was $140.0 \pm 4.8$ months. The mean survival time was $71.1 \pm 4.1$ months while the median survival time was $40.0 \pm 6.7$ months. In addition, the 3 - and 5-year OS rate was $46.9 \%$ and $37.4 \%$, respectively (Figure S2).

Pre-surgery factors such as age $(\mathrm{p}=0.005)$, cell differentiation $(p=0.011)$, BMI $(p=0.011)$, total protein $(T P$, $\mathrm{p}=0.024), \mathrm{A} / \mathrm{G}(\mathrm{p}=0.073)$, total bilirubin (TBIL, $\mathrm{p}=0.081)$, direct bilirubin (DBIL, $\mathrm{p}=0.115)$, HDL-C $(\mathrm{p}<0.001)$, LDL-C $(\mathrm{p}=0.045)$, serum potassium $(\mathrm{p}=0.105)$, serum calcium $(p=0.147)$, or serum magnesium $(p=0.049)$ were significantly associated with the GC prognosis in the univariate analysis. On the other hand, post-surgery factors such as $\mathrm{T} \quad(\mathrm{p}=0.008), \mathrm{N} \quad(\mathrm{p}=0.031), \mathrm{M} \quad(\mathrm{p}=0.014)$, or TNM $(p=0.052)$ were shown to be significantly associated with the GC prognosis in the univariate analysis (Table 2). Unlike the univariate data, multivariate analysis showed a slight deviation. Pre-surgery factors such as age (Hazard ratio, $[\mathrm{HR}]=1.022, \quad \mathrm{p}<0.001)$, poor cell differentiation $(\mathrm{HR}=1.618, \mathrm{p}<0.001)$, HDL-C levels of more than 1.04 $\mathrm{mmol} / \mathrm{L} \quad(\mathrm{HR}=0.430, \mathrm{p}<0.001)$, BMI score of more than $24 \mathrm{~kg} / \mathrm{m}^{2}(\mathrm{HR}=0.241, \mathrm{p}<0.001)$, serum magnesium level at the range of $0.75-1.02 \mathrm{mmol} / \mathrm{L}(\mathrm{HR}=0.651, \mathrm{p}=0.021)$ were independent prognostic indicators. There was no significant difference between serum potassium levels and OS $(\mathrm{p}=0.282)$. TNM $(\mathrm{HR}=2.734, \mathrm{p}<0.001)$ was the only postsurgery independent prognostic factor in the multivariate analysis (Table 2). 
Table I Preoperative and Postoperative Characteristics of 303 Gastric Cancer Patients

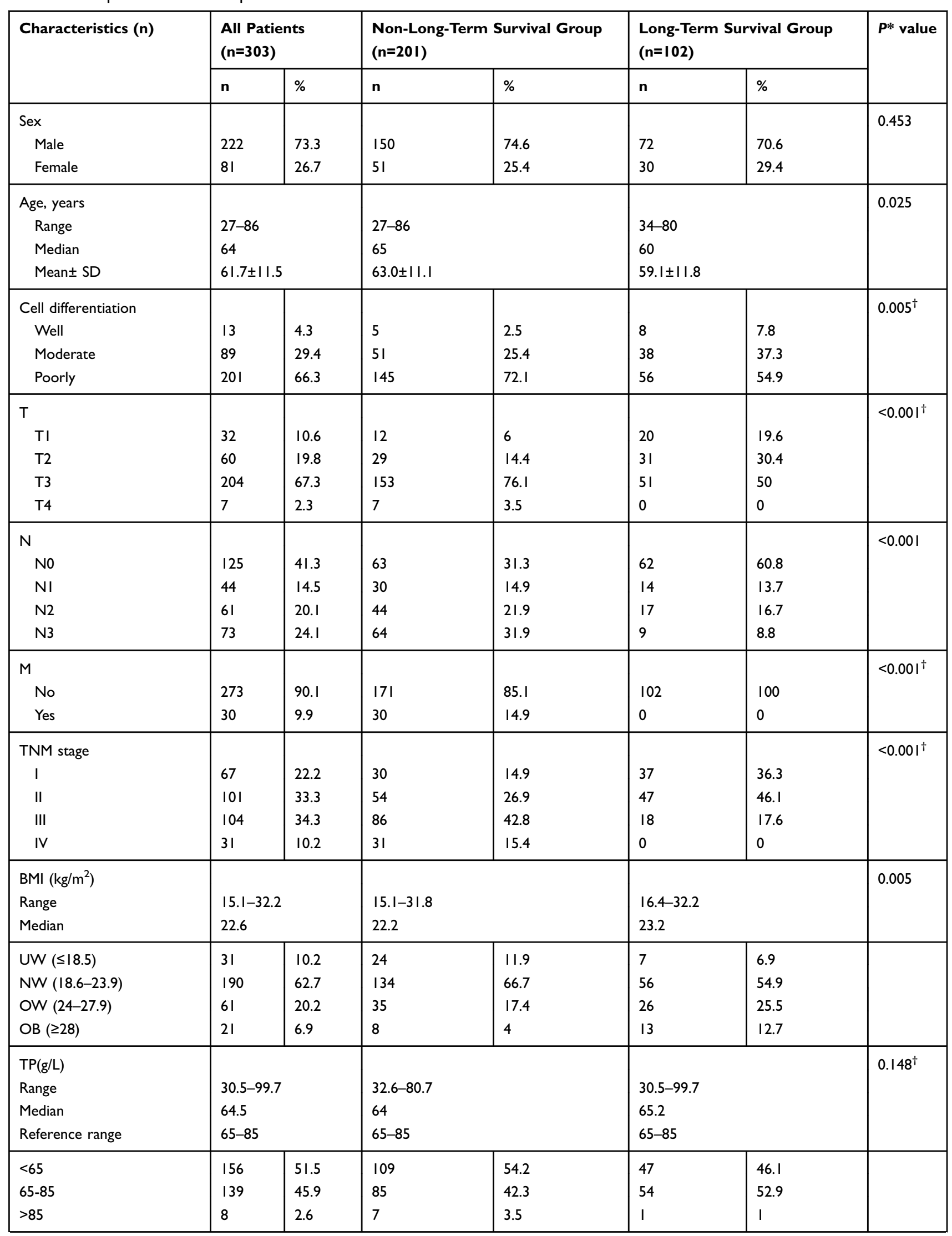

(Continued) 
Table I (Continued).

\begin{tabular}{|c|c|c|c|c|c|c|c|}
\hline \multirow[t]{2}{*}{ Characteristics (n) } & \multicolumn{2}{|c|}{$\begin{array}{l}\text { All Patients } \\
(n=303)\end{array}$} & \multicolumn{2}{|c|}{$\begin{array}{l}\text { Non-Long-Term Survival Group } \\
(n=201)\end{array}$} & \multicolumn{2}{|c|}{$\begin{array}{l}\text { Long-Term Survival Group } \\
(n=102)\end{array}$} & \multirow[t]{2}{*}{$P *$ value } \\
\hline & $\mathbf{n}$ & $\%$ & $\mathbf{n}$ & $\%$ & $\mathbf{n}$ & $\%$ & \\
\hline Alb (g/L) & \multirow{2}{*}{\multicolumn{2}{|c|}{$203-497$}} & \multirow{2}{*}{\multicolumn{2}{|c|}{$20.3-49.7$}} & & 0.003 \\
\hline Range & & & & & 30 & & \\
\hline Median & \multicolumn{2}{|c|}{39} & \multicolumn{2}{|c|}{38.5} & \multicolumn{2}{|c|}{40.9} & \\
\hline Reference range & \multicolumn{2}{|c|}{$40-55$} & \multicolumn{2}{|l|}{$40-55$} & \multicolumn{2}{|c|}{$40-55$} & \\
\hline$<40$ & 175 & 57.8 & 128 & 63.7 & 47 & 46.1 & \\
\hline$\geq 40$ & 128 & 42.2 & 73 & 36.3 & 55 & 53.9 & \\
\hline $\mathrm{A} / \mathrm{G}$ & & \multirow{2}{*}{\multicolumn{2}{|c|}{$0.6-2.7$}} & \multirow{2}{*}{\multicolumn{2}{|c|}{$1.1-2.3$}} & $0.012^{\dagger}$ \\
\hline Range & & & & & & & \\
\hline Median & \multicolumn{2}{|c|}{$0.6-2.7$} & \multicolumn{2}{|c|}{1.5} & \multicolumn{2}{|c|}{1.6} & \\
\hline Reference range & \multicolumn{2}{|c|}{$1.2-2.4$} & & & \\
\hline$<1.2$ & 27 & 8.9 & 24 & 11.9 & 3 & 2.9 & \\
\hline I.2-2.4 & 275 & 90.8 & 176 & 87.6 & 99 & 97.1 & \\
\hline$>2.4$ & 1 & 0.3 & 1 & 0.5 & 0 & 0 & \\
\hline Glu (mmol/L) & & & & & & & $0.014^{\dagger}$ \\
\hline Range & $2.1-1$ & & $2.1-12$ & & $4-1$ & & \\
\hline Median & 5 & & 4.9 & & 5.2 & & \\
\hline Reference range & $3.9-6$ & & $3.9-6$. & & 3.9 & & \\
\hline$<3.9$ & 12 & 4 & 12 & 6 & 0 & 0 & \\
\hline $3.9-6.1$ & 240 & 79.2 & 159 & 79.1 & 81 & 79.4 & \\
\hline$>6.1$ & 51 & 16.8 & 30 & 14.9 & 21 & 20.6 & \\
\hline TBIL (umol/L) & & & & & & & 0.116 \\
\hline Range & $4.8-4$ & & $4.8-43$ & & $5.1-$ & & \\
\hline Median & 12 & & 12.3 & & 11.8 & & \\
\hline Reference range & $0-23$ & & $0-23$ & & $0-2$ & & \\
\hline $0-23$ & 285 & 94.1 & 186 & 92.5 & 99 & 97.1 & \\
\hline$>23$ & 18 & 4.6 & 15 & 7.5 & 3 & 2.9 & \\
\hline DBIL (umol/L) & & & & & & & $0.397^{\dagger}$ \\
\hline Range & $1-19$ & & $1-19.7$ & & I.I- & & \\
\hline Median & 3.7 & & 3.7 & & 3.6 & & \\
\hline Reference range & $0-8$ & & $0-8$ & & $0-8$ & & \\
\hline $0-8$ & 289 & 95.4 & 190 & 94.5 & 99 & 97.1 & \\
\hline$>8$ & 14 & 4.6 & 11 & 5.5 & 3 & 2.9 & \\
\hline TC (mmol/L) & & & & & & & 0.043 \\
\hline Range & $2-10$ & & $2.1-5.8$ & & $2-1$ & & \\
\hline Median & 4 & & 4 & & 4.3 & & \\
\hline Reference range & $0-5.1$ & & $0-5.18$ & & $0-5$ & & \\
\hline $0-5.18$ & 285 & 94.1 & 193 & 96 & 92 & 90.2 & \\
\hline$>5.18$ & 18 & 5.9 & 8 & 4 & 10 & 9.8 & \\
\hline TG (mmol/L) & & & & & & & 0.137 \\
\hline Range & $0-7.6$ & & $0-5.9$ & & 0.2 & & \\
\hline Median & I.I & & I & & 1.2 & & \\
\hline Reference range & $0-1.7$ & & $0-1.7$ & & $0-1$ & & \\
\hline
\end{tabular}

(Continued) 
Table I (Continued).

\begin{tabular}{|c|c|c|c|c|c|c|c|}
\hline \multirow[t]{2}{*}{ Characteristics (n) } & \multicolumn{2}{|c|}{$\begin{array}{l}\text { All Patients } \\
(n=303)\end{array}$} & \multicolumn{2}{|c|}{$\begin{array}{l}\text { Non-Long-Term Survival Group } \\
(n=201)\end{array}$} & \multicolumn{2}{|c|}{$\begin{array}{l}\text { Long-Term Survival Group } \\
(n=102)\end{array}$} & \multirow[t]{2}{*}{$P *$ value } \\
\hline & $\mathbf{n}$ & $\%$ & $\mathbf{n}$ & $\%$ & $\mathbf{n}$ & $\%$ & \\
\hline $0-1.7$ & 254 & 83.8 & 173 & 86.1 & 81 & 79.4 & \\
\hline$>1.7$ & 49 & 16.2 & 28 & 13.9 & 21 & 20.6 & \\
\hline HDL-C (mmol/L) & & \multirow{2}{*}{\multicolumn{2}{|c|}{$0.4-9.5$}} & & $<0.001$ \\
\hline Range & \multirow{2}{*}{\multicolumn{2}{|c|}{$\begin{array}{l}0.4-9.9 \\
1.6\end{array}$}} & & & $0.5-$ & & \\
\hline Median & & & \multicolumn{2}{|c|}{1.7} & \multicolumn{2}{|c|}{1.6} & \\
\hline Reference range & \multicolumn{2}{|c|}{$0-1.04$} & \multicolumn{2}{|c|}{$0-1.04$} & \multicolumn{2}{|c|}{$0-1.04$} & \\
\hline $0-1.04$ & 120 & 39.6 & 94 & 46.8 & 26 & 25.5 & \\
\hline$>1.04$ & 183 & 60.4 & 107 & 53.2 & 76 & 74.5 & \\
\hline LDL-C (mmol/L) & \multirow{2}{*}{\multicolumn{2}{|c|}{$0.1-4.8$}} & \multirow{2}{*}{\multicolumn{2}{|c|}{$0.1-4.2$}} & \multirow{2}{*}{\multicolumn{2}{|c|}{ I.I-4.8 }} & 0.011 \\
\hline Range & & & & & & & \\
\hline Median & \multicolumn{2}{|c|}{2.4} & \multicolumn{2}{|c|}{2.3} & \multicolumn{2}{|c|}{2.6} & \\
\hline Reference range & \multicolumn{2}{|c|}{$0-3.37$} & \multicolumn{2}{|c|}{$0-3.37$} & \multicolumn{2}{|c|}{$0-3.37$} & \\
\hline $0-3.37$ & 285 & 94.1 & 194 & 96.5 & 91 & 89.2 & \\
\hline$>3.37$ & 18 & 5.9 & 7 & 3.5 & 11 & 10.8 & \\
\hline Serum sodium(mmol/L) & & & & & & & $0.023^{\dagger}$ \\
\hline Range & 104.6 & & 124 & & 104 & & \\
\hline Median & $14 \mid .3$ & & $14 \mid$ & & $|4|$ & & \\
\hline Reference range & $137-$ & & $137-$ & & 137 & & \\
\hline$<137$ & 36 & 11.9 & 27 & 13.4 & 9 & 8.8 & \\
\hline |37-147 & 261 & 86.1 & 170 & 84.6 & 91 & 89.2 & \\
\hline$>147$ & 6 & 2 & 4 & 2 & 2 & 2 & \\
\hline Serum potassium (mmol/L) & & & & & & & $0.182^{\dagger}$ \\
\hline Range & $2.5-5$ & & $2.5-5$ & & $3-5$ & & \\
\hline Median & 4.1 & & 4.1 & & 4.1 & & \\
\hline Reference range & $3.5-5$ & & $3.5-5$ & & $3.5-$ & & \\
\hline$<3.5$ & 26 & 8.6 & 21 & 10.4 & 5 & 4.9 & \\
\hline $3.5-5.3$ & 271 & 89.4 & 175 & 87.1 & 96 & 94.1 & \\
\hline$>5.3$ & 6 & 2 & 5 & 2.5 & 1 & 1 & \\
\hline Serum chlorine $(\mathrm{mmol} / \mathrm{L})$ & & & & & & & $0.344^{\dagger}$ \\
\hline Range & 77.8 & & $77.8-$ & & 92. & & \\
\hline Median & 101.5 & & 102 & & 101 & & \\
\hline Reference range & I & & I & & 99 & & \\
\hline$<99$ & 47 & 15.5 & 35 & 17.4 & 12 & 11.8 & \\
\hline $99-110$ & 250 & 82.5 & 161 & 80.1 & 89 & 87.3 & \\
\hline$>110$ & 6 & 2 & 5 & 2.5 & 1 & I & \\
\hline Serum calcium (mmol/L) & & & & & & & 0.659 \\
\hline Range & $1.7-3$ & & $1.7-2$ & & 1.9 & & \\
\hline Median & 2.3 & & 2.3 & & 2.3 & & \\
\hline Reference range & $2.11-$ & & $2.11-$ & & 2.1 & & \\
\hline$<2.11$ & 24 & 7.9 & 17 & 8.5 & 7 & 6.9 & \\
\hline $2.11-2.52$ & 252 & 83.2 & 168 & 83.6 & 84 & 82.4 & \\
\hline$>2.52$ & 27 & 8.9 & 16 & 8 & 11 & 10.8 & \\
\hline
\end{tabular}

(Continued) 
Table I (Continued).

\begin{tabular}{|c|c|c|c|c|c|c|c|}
\hline \multirow[t]{2}{*}{ Characteristics (n) } & \multicolumn{2}{|c|}{$\begin{array}{l}\text { All Patients } \\
(n=303)\end{array}$} & \multicolumn{2}{|c|}{$\begin{array}{l}\text { Non-Long-Term Survival Group } \\
(n=201)\end{array}$} & \multicolumn{2}{|c|}{$\begin{array}{l}\text { Long-Term Survival Group } \\
(n=102)\end{array}$} & \multirow[t]{2}{*}{$P *$ value } \\
\hline & $\mathbf{n}$ & $\%$ & $\mathbf{n}$ & $\%$ & $\mathbf{n}$ & $\%$ & \\
\hline $\begin{array}{l}\text { Serum phosphorus (mmol/L) } \\
\text { Range } \\
\text { Median } \\
\text { Reference range }\end{array}$ & \multicolumn{2}{|c|}{$\begin{array}{l}0.1-5.1 \\
I \\
0.85-\mid .5 I\end{array}$} & \multicolumn{2}{|c|}{$\begin{array}{l}0.1-5.1 \\
I \\
0.85-\mid .5 I\end{array}$} & \multicolumn{2}{|c|}{$\begin{array}{l}0-1.9 \\
1.1 \\
0.85-1.51\end{array}$} & $0.27 I^{\dagger}$ \\
\hline $\begin{array}{l}<0.85 \\
0.85-|.5| \\
>1.5 \mid\end{array}$ & $\begin{array}{l}60 \\
233 \\
10\end{array}$ & $\begin{array}{l}19.8 \\
76.9 \\
3.3\end{array}$ & $\begin{array}{l}45 \\
149 \\
7\end{array}$ & $\begin{array}{l}22.4 \\
74.1 \\
3.5\end{array}$ & $\begin{array}{l}15 \\
84 \\
3\end{array}$ & $\begin{array}{l}14.7 \\
82.4 \\
2.9\end{array}$ & \\
\hline $\begin{array}{l}\text { Serum magnesium }(\mathrm{mmol} / \mathrm{L}) \\
\text { Range } \\
\text { Median } \\
\text { Reference range }\end{array}$ & \multicolumn{2}{|c|}{$\begin{array}{l}0.5-2 \\
0.9 \\
0.75-1.02\end{array}$} & \multicolumn{2}{|c|}{$\begin{array}{l}0.5-2 \\
0.8 \\
0.75-1.02\end{array}$} & \multicolumn{2}{|c|}{$\begin{array}{l}0.6-1.1 \\
0.9 \\
0.75-1.02\end{array}$} & $0.136^{\dagger}$ \\
\hline $\begin{array}{l}<0.75 \\
0.75-1.02 \\
>1.02\end{array}$ & $\begin{array}{l}45 \\
245 \\
13\end{array}$ & $\begin{array}{l}14.9 \\
80.9 \\
4.3\end{array}$ & $\begin{array}{l}34 \\
156 \\
11\end{array}$ & $\begin{array}{l}16.9 \\
77.6 \\
5.5\end{array}$ & $\begin{array}{l}11 \\
89 \\
2\end{array}$ & $\begin{array}{l}10.8 \\
87.3 \\
2\end{array}$ & \\
\hline
\end{tabular}

Notes: Date are presented as $\mathrm{n}(\%)$, mean \pm standard deviation, or median; * the data is compared using $\chi 2$ test; ${ }^{\dagger}$ Fisher's exact test.

Abbreviations: T, tumor invasion; N, lymph node metastasis; M, distant metastasis; BMI, body mass index; UW, underweight; NW, normal weight; OW, overweight; OB, obesity; TP, total protein; Alb, albumin; A/G, albumin: globulin; Glu, blood glucose; TBIL, total bilirubin; DBIL, direct bilirubin; TC, total cholesterol; TG, triglyceride; HDL-C, high-density lipoprotein cholesterol; LDL-C, low-density lipoprotein cholesterol.

Although there was no significant association between sex and GC prognosis in the univariate or multivariate analysis, some studies showed that sex affected the prognosis. ${ }^{18}$ Besides, some studies showed that electrolyte imbalance, especially hypokalemia, is common in patients who received abdominal surgery, affecting the patients' postoperative recovery. ${ }^{19}$ Thus, serum potassium and sex were incorporated into the prediction model.

\section{Establishment of a Nomogram for Predicting 5-year OS}

For the development of the nomogram (Figure 1), we incorporated clinical features and blood biochemical parameters (age, sex, cell differentiation, BMI, HDL-C, serum potassium, serum magnesium) as defined by the multivariate analysis.

Figure 1 shows the nomogram: vertical lines are drawn from the correct status of each prognostic factor to the top axis (points). After the addition of all the points, a vertical line was drawn from the "total points" axis to the bottom axes. This helps in the conversion into a 5-year survival probability.

Figure 2 depicts the Time-dependent AUC. The learned Cox model resulted in AUC ranging from 0.757 to 0.802 for different time points. Additionally, the sensitivity and specificity for predicting 5-year survival rate at different cutoff values are summarized in Table 3. At a cutoff value of $>0.70$, specificity was $78.95 \%$ while sensitivity was $63.46 \%$. Although higher cutoff values result in higher specificity, sensitivity falls rapidly.

\section{Validation of the Nomogram for the Prediction of a 5-year OS}

The 5-year OS predictive accuracy of the nomogram was 0.701 in the internal validation (500 bootstraps). The 5 -year OS prediction accuracy was validated by the calibration curve which showed a correlation between the actual observed outcome and the prediction by the nomogram. This correlation data from the calibration curve was observed even when the nomogram prediction probability was less than 20\% (Figure 3A).

\section{Comparison of the Nomogram with TNM Prognostic Indexes}

We then compared the model with the 8th AJCC TNM staging system for the 5 -year OS after the radical gastrectomy. Our findings showed that the nomogram displayed higher levels of accuracy over the TNM staging (C-index, 0.701, 95\% confidence interval [CI], 
Table 2 Univariate and Multivariable Analyses of Risk Factors Associated with the Prognosis of Gastric Cancer

\begin{tabular}{|c|c|c|c|c|c|c|c|}
\hline \multirow{2}{*}{\multicolumn{2}{|c|}{ Clinicopathological Parameters }} & \multicolumn{2}{|c|}{$\begin{array}{l}\text { Cox Univariate } \\
\text { Regression Model } \\
\text { Analysis }\end{array}$} & \multirow{3}{*}{$\begin{array}{l}P \text { values } \\
\\
0.005 \\
0.662 \\
0.011\end{array}$} & \multicolumn{2}{|c|}{$\begin{array}{l}\text { Cox Multivariable } \\
\text { Regression Model } \\
\text { Analysis }\end{array}$} & \multirow{3}{*}{$\begin{array}{l}P \text { values } \\
<0.001 \\
<0.001\end{array}$} \\
\hline & & \multirow{2}{*}{$\begin{array}{l}\text { HR } \\
1.019 \\
1.085 \\
1.509\end{array}$} & \multirow{2}{*}{$\begin{array}{l}(95 \% \mathrm{Cl}) \\
1.006-1.033 \\
0.754-1.561 \\
1.098-2.073\end{array}$} & & \multirow{2}{*}{$\begin{array}{l}\mathbf{H R} \\
1.022 \\
1.618\end{array}$} & \multirow{2}{*}{$\begin{array}{l}(95 \% \mathrm{Cl}) \\
1.009-1.035 \\
1.226-2.136\end{array}$} & \\
\hline Markers before surgery & $\begin{array}{l}\text { Age } \\
\text { Sex (female vs male) } \\
\text { Cell differentiation (Poorly vs Moderate+ Well) }\end{array}$ & & & & & & \\
\hline & $\begin{array}{l}\text { BMI }\left(\mathrm{kg} / \mathrm{m}^{2}\right) \\
18.6-23.9 \text { vs } \leq 18.5 \\
24-27.9 \text { vs } \leq 18.5 \\
>28 \text { vs } \leq 18.5\end{array}$ & $\begin{array}{l}0.870 \\
0.552 \\
0.353\end{array}$ & $\begin{array}{l}0.542-1.396 \\
0.316-0.963 \\
0.158-0.789\end{array}$ & $\begin{array}{l}0.565 \\
0.036 \\
0.011\end{array}$ & $\begin{array}{l}0.665 \\
0.420 \\
0.241\end{array}$ & $\begin{array}{l}0.421-1.050 \\
0.245-0.720 \\
0.110-0.533\end{array}$ & $\begin{array}{l}0.078 \\
0.002 \\
<0.001\end{array}$ \\
\hline & $\begin{array}{l}\mathrm{TP}(\mathrm{g} / \mathrm{L}) \\
65-85 \text { vs }<65 \\
>85 \text { vs }<65\end{array}$ & $\begin{array}{l}0.877 \\
2.694\end{array}$ & $\begin{array}{l}0.643-1.197 \\
1.14|-6.36|\end{array}$ & $\begin{array}{l}0.408 \\
0.024\end{array}$ & & & \\
\hline & Alb $(<40$ vs $\geq 40 \mathrm{~g} / \mathrm{L})$ & 1.040 & $0.696-1.555$ & 0.849 & & & \\
\hline & $\begin{array}{l}\mathrm{A} / \mathrm{G} \\
\mathrm{I} .2-2.4 \text { vs }<1.2 \\
>2.4 \text { vs }<1.2\end{array}$ & $\begin{array}{l}0.764 \\
0.091\end{array}$ & $\begin{array}{l}0.462-1.262 \\
0.007-1.245\end{array}$ & $\begin{array}{l}0.293 \\
0.073\end{array}$ & & & \\
\hline & $\begin{array}{l}\text { Glu }(\mathrm{mmol} / \mathrm{L}) \\
3.9-6.1 \text { vs }<3.9 \\
>6.1 \text { vs }<3.9\end{array}$ & $\begin{array}{l}0.757 \\
0.668\end{array}$ & $\begin{array}{l}0.374-1.534 \\
0.304-1.47 \mid\end{array}$ & $\begin{array}{l}0.440 \\
0.317\end{array}$ & & & \\
\hline & $\begin{array}{l}\text { TBIL (> } 23 \text { vs.0-23 umol/L) } \\
\text { DBIL (> } 8 \text { vs.0-8 umol/L) } \\
\text { TC (>5.18 vs } 0-5.18 \mathrm{mmol} / \mathrm{L}) \\
\text { TG (> I.7 vs } 0-1.7 \mathrm{mmol} / \mathrm{L}) \\
\text { HDL-C (> } 1.04 \text { vs } 0-1.04 \mathrm{mmol} / \mathrm{L}) \\
\text { LDL-C (> } 1.7 \text { vs } 0-1.7 \mathrm{mmol} / \mathrm{L})\end{array}$ & $\begin{array}{l}2.786 \\
0.340 \\
1.367 \\
0.869 \\
0.512 \\
0.474\end{array}$ & $\begin{array}{l}0.880-8.814 \\
0.089-1.302 \\
0.545-3.430 \\
0.524-1.441 \\
0.377-0.695 \\
0.228-0.983\end{array}$ & $\begin{array}{l}0.081 \\
0.115 \\
0.506 \\
0.587 \\
<0.001 \\
0.045\end{array}$ & 0.430 & $0.321-0.574$ & $<0.001$ \\
\hline & $\begin{array}{l}\text { Serum sodium(mmol/L) } \\
\text { |37-147 vs }<137 \\
>147 \text { vs }<137\end{array}$ & $\begin{array}{l}0.693 \\
0.418\end{array}$ & $\begin{array}{l}0.400-1.199 \\
0.122-1.430\end{array}$ & $\begin{array}{l}0.190 \\
0.164\end{array}$ & & & \\
\hline & $\begin{array}{l}\text { Serum potassium }(\mathrm{mmol} / \mathrm{L}) \\
3.5-5.3 \text { vs }<3.5 \\
>5.3 \text { vs }<3.5\end{array}$ & $\begin{array}{l}0.708 \\
2.419\end{array}$ & $\begin{array}{l}0.420-1.192 \\
0.832-7.027\end{array}$ & $\begin{array}{l}0.194 \\
0.105\end{array}$ & $\begin{array}{l}0.695 \\
1.646\end{array}$ & $\begin{array}{l}0.448-1.079 \\
0.664-4.078\end{array}$ & $\begin{array}{l}0.105 \\
0.282\end{array}$ \\
\hline & $\begin{array}{l}\text { Serum chlorine }(\mathrm{mmol} / \mathrm{L}) \\
99-110 \text { vs }<99 \\
>110 \text { vs }<99\end{array}$ & $\begin{array}{l}1.038 \\
1.611\end{array}$ & $\begin{array}{l}0.627-1.717 \\
0.513-5.060\end{array}$ & $\begin{array}{l}0.886 \\
0.414\end{array}$ & & & \\
\hline & $\begin{array}{l}\text { Serum calcium }(\mathrm{mmol} / \mathrm{L}) \\
2.1 \mathrm{I}-2.52 \text { vs }<2.1 \mathrm{I} \\
>2.52 \text { vs }<2.1 \mathrm{I}\end{array}$ & $\begin{array}{l}1.478 \\
0.993\end{array}$ & $\begin{array}{l}0.87 I-2.506 \\
0.463-2.130\end{array}$ & $\begin{array}{l}0.147 \\
0.986\end{array}$ & & & \\
\hline & $\begin{array}{l}\text { Serum phosphorus }(\mathrm{mmol} / \mathrm{L}) \\
0.85-1.5 \mathrm{I} \text { vs }<0.85 \\
>2.52 \text { vs }<2.11\end{array}$ & $\begin{array}{l}0.973 \\
1.070\end{array}$ & $\begin{array}{l}0.660-1.434 \\
0.460-2.490\end{array}$ & $\begin{array}{l}0.888 \\
0.875\end{array}$ & & & \\
\hline & $\begin{array}{l}\text { Serum magnesium }(\mathrm{mmol} / \mathrm{L}) \\
0.75-1.02 \text { vs }<0.75 \\
>1.02 \text { vs }<0.75\end{array}$ & $\begin{array}{l}0.679 \\
1.547\end{array}$ & $\begin{array}{l}0.463-0.998 \\
0.759-3.154\end{array}$ & $\begin{array}{l}0.049 \\
0.230\end{array}$ & $\begin{array}{l}0.651 \\
1.630\end{array}$ & $\begin{array}{l}0.45 I-0.938 \\
0.815-3.260\end{array}$ & $\begin{array}{l}0.021 \\
0.167\end{array}$ \\
\hline
\end{tabular}

(Continued) 
Table 2 (Continued).

\begin{tabular}{|c|c|c|c|c|c|c|c|}
\hline \multirow{2}{*}{\multicolumn{2}{|c|}{ Clinicopathological Parameters }} & \multicolumn{2}{|c|}{$\begin{array}{l}\text { Cox Univariate } \\
\text { Regression Model } \\
\text { Analysis }\end{array}$} & \multirow[t]{2}{*}{$P$ values } & \multicolumn{2}{|c|}{$\begin{array}{l}\text { Cox Multivariable } \\
\text { Regression Model } \\
\text { Analysis }\end{array}$} & \multirow[t]{2}{*}{$P$ values } \\
\hline & & HR & $(95 \% \mathrm{Cl})$ & & HR & $(95 \% \mathrm{Cl})$ & \\
\hline Markers after surgery & $\begin{array}{l}\text { Invasion depth (T3+T4 vs } \mathrm{TI}+\mathrm{T} 2) \\
\text { Lymph node metastasis (YES vs NO) } \\
\text { Distant metastases (YES vs NO) } \\
\text { Clinical staging (III+IV vs I+II) }\end{array}$ & $\begin{array}{l}1.713 \\
1.549 \\
1.755 \\
1.548\end{array}$ & $\begin{array}{l}1.150-2.55 \mid \\
1.041-2.306 \\
1.120-2.750 \\
0.996-2.406\end{array}$ & $\begin{array}{l}0.008 \\
0.031 \\
0.014 \\
0.052\end{array}$ & 2.734 & $2.027-3.690$ & $<0.001$ \\
\hline
\end{tabular}

Note: Cell differentiation was from WHO histological type.

Abbreviations: $\mathrm{HR}$, hazard ratio; $\mathrm{Cl}$, confidence interval; vs, versus.

\subsection{9-0.733 vs $0.666,95 \%$ CI, $0.630-0.702 ; p=0.016)$ Discussion}

(Table S1). Therefore, the nomogram is an accurate tool and can be adopted to predict GC prognosis before surgery. The DCA for the model is as shown in Figure 3B.

This study has demonstrated that age, sex, cell differentiation, HDL-C, BMI, serum potassium or serum magnesium are useful bio-indicators in terms of predicting a 5-year OS for GC patients after receiving radical gastrectomy. The

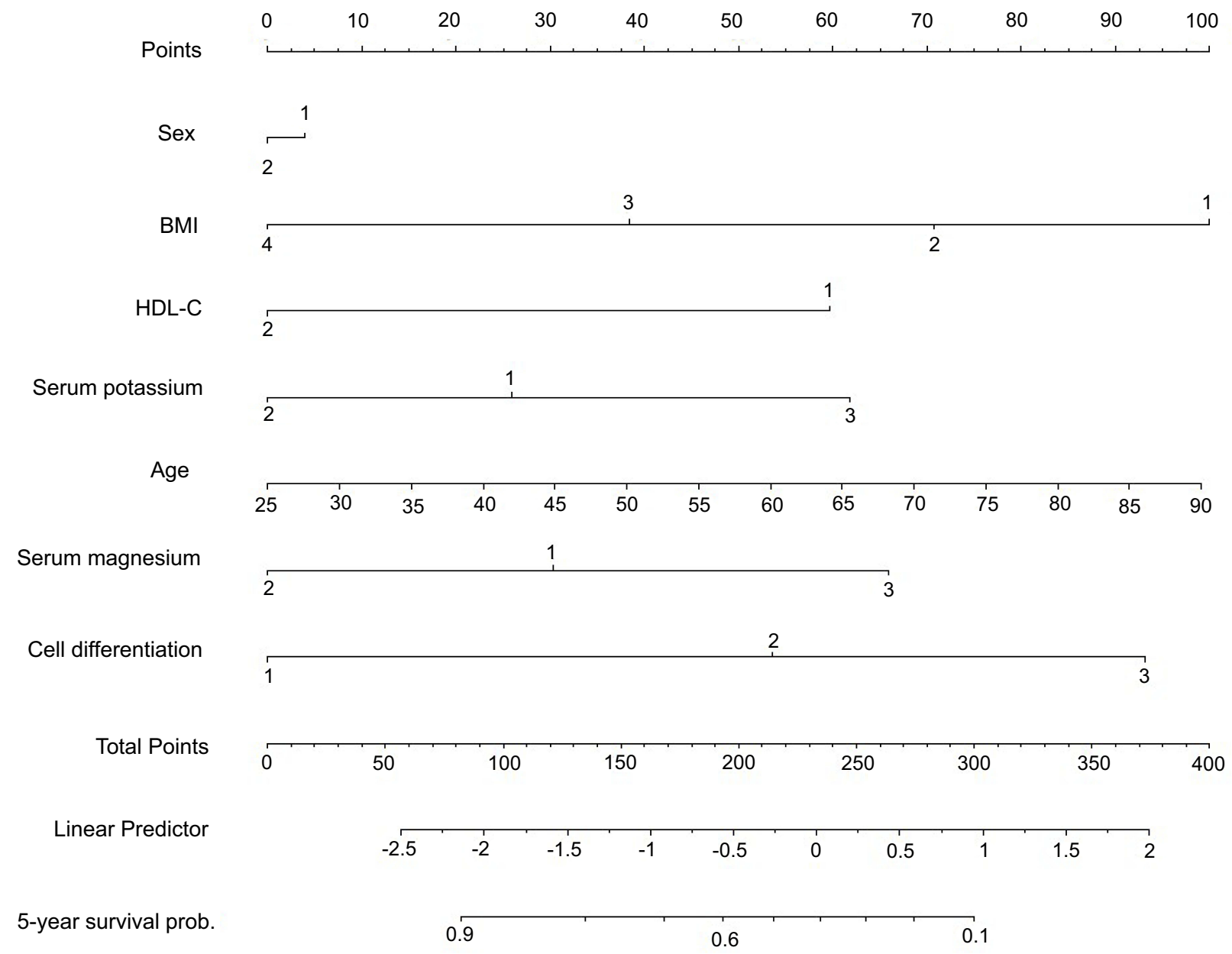

Figure I Nomogram to predict individual patient-level 5-year overall survival based on preoperative clinical biochemical features. The value of an individual patient is located on each variable axis, and predictor points ("Points" scale; top) correspond to each variable. Sum of all the seven variables is located on the total point axis.

Abbreviations: BMI, body mass index; HDL-C, high-density lipoprotein cholesterol; prob, probability. 
Time-dependent AUC

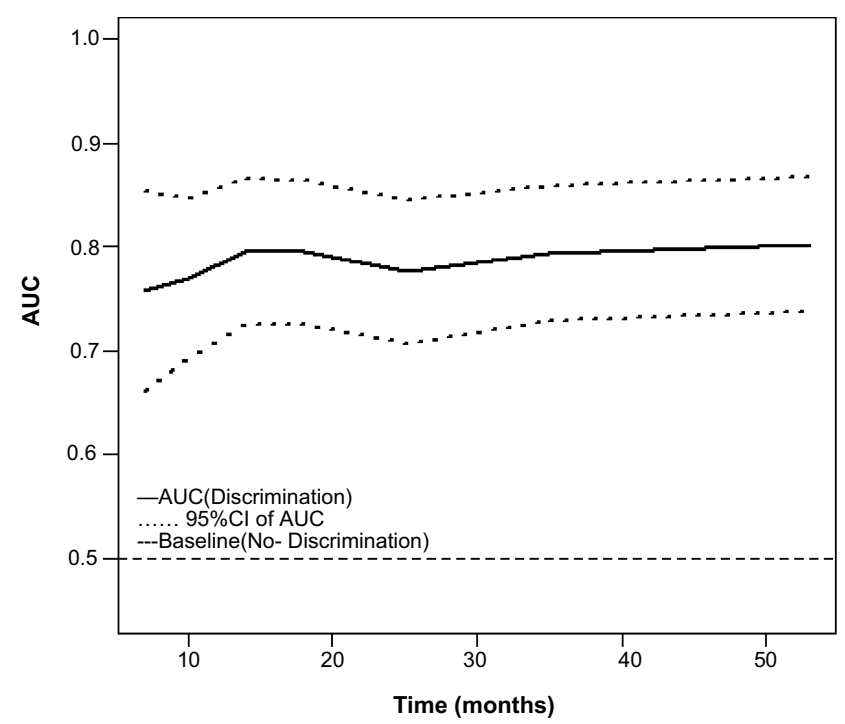

Figure 2 Time-dependent AUC. The solid and dashed lines depict the AUC and random chance, respectively. The dotted line shows the $95 \% \mathrm{Cl}$ of AUC. The AUC increases with the number of months.

Abbreviation: $\mathrm{Cl}=$ confidence interval.

combination of all the seven pre-surgery parameters has resulted in a C-index of 0.701 , suggesting this combination of bio-indicators may act as an independent factor capable of predicting prognosis for GC patients. Besides predicting disease progression, it may also be used to elaborate surgical strategies and estimate possible postoperative survival prior to surgery. Unlike the nomogram, classic TNM staging is only possible in predicting survival after surgery. We have introduced a nomogram based on patients' basic clinical features and preoperative biochemical indicators. The nomogram predicts the 5-year OS among GC patients (Figure 1) with a reliable performance (AUC of 0.78). This nomogram may comprehend treatment and followup plans for patients with GC. Essentially, our study incorporates the preoperative blood biochemical indicators to predict outcomes of GC patients. Although studies have reported that some molecular markers predict disease prognosis and survival for GC patients, most of these molecular tools require professional testing, are timeconsuming and expensive and, therefore, limit their usefulness in clinical settings at large. The blood biochemical indicators used here are readily tested, non-invasive or minimally invasive and inexpensive, thus, may serve as a preferred option in most clinical settings.

Our nomogram analysis has shown that age is an independent and baseline predictor for the OS. Patients with advanced age who underwent radical gastrectomy due to GC have been associated with a low 5-year survival rate, in keeping with previous reports. ${ }^{20}$ The phenomenon may be attributed to comorbidities, compromised immunity, and malnutrition amongst others in senior patients. In addition, the low survival rate in older patients has been associated with a lack of tumor-related early symptoms as well as routine screening, thus, late-stage diagnosis and delayed treatment.

Previous studies, including ours, have shown that BMIdefined underweight is correlated with poor GC prognosis. ${ }^{21,22}$ This study has shown that GC patients with higher BMI are correlated with better survival rates, confirming previous findings. It is hypothesized that lower BMI may be associated with difficulties in food intake due to late-stage AGC; thus, rapid weight loss indicates a deterioration of general health in GC patients. Furthermore, a low BMI prior to surgery renders a patient difficulty to meet perioperative nutritional requirements which, in turn, affect postoperative recovery, leading to poor prognosis.

Interestingly, unlike multivariable analysis, our univariate analysis has shown that serum calcium appears to affect the prognosis of GC patients. LIPKIN et al have observed that serum calcium supplementation can reduce

Table 3 Values of Sensitivity, Specificity, and Predictive Values of the Nomogram Scores at Different Cutoff Values

\begin{tabular}{|l|l|l|l|l|l|}
\hline Nomogram Score/Predicted Probability & Sensitivity (\%) & Specificity (\%) & Accuracy (\%) & PPV (\%) & NPV (\%) \\
\hline$\geq 0.20$ & 95.19 & 16.84 & 70.63 & 71.48 & 61.54 \\
$\geq 0.30$ & 94.71 & 16.84 & 70.30 & 71.38 & 59.26 \\
$\geq 0.40$ & 79.81 & 52.63 & 71.29 & 78.67 & 54.35 \\
$\geq 0.50$ & 83.65 & 44.21 & 71.29 & 76.65 & 55.26 \\
$\geq 0.60$ & 81.25 & 50.53 & 71.62 & 78.24 \\
$\geq 0.70$ & 63.46 & 78.95 & 68.32 & 55.17 \\
$\geq 0.80$ & 63.46 & 80.00 & 68.65 & 86.84 & 49.67 \\
\hline
\end{tabular}

Notes: The predicted probability/nomogram score is a numeric value representing the prediction model score of the individual patient. Abbreviations: NPV, negative predictive value; PPV, positive predictive value. 
A

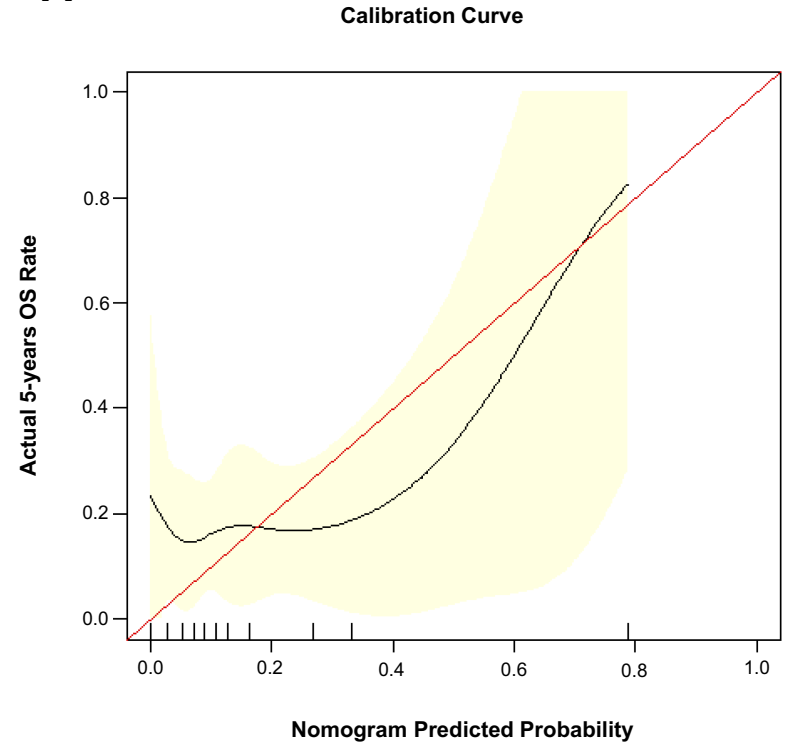

B

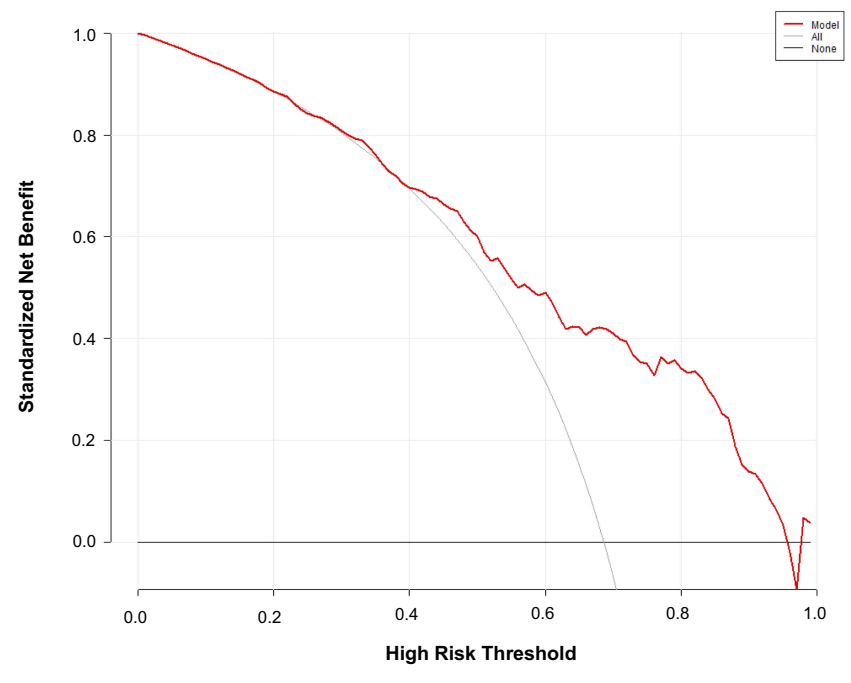

Figure 3 (A) The Calibration curves of 5-year overall survival nomogram. The red line is reference line, and the black line is fitting. The yellow area represents the $95 \% \mathrm{Cl}$. After 500 repetitions of bootstrap, the calibration curves showed a good correlation between the predicted probability and actual probability. (B) Decision curve analysis for the 5-year overall survival nomogram. Solid bold line: the assumption that no patients will experience the event; solid thin line: the assumption that all patients will relapse; red line: net benefit of a strategy of treating patients according to the nomogram predictions.

the chances of colon or esophagus cancer in experimental animals. ${ }^{23}$ LIN et al however, have shown that there are no significant differences in serum calcium levels between GC patients and non-GC patients. ${ }^{24}$ To date, it has not been reported that serum calcium levels are correlated with the prognosis of GC patients. Furthermore, normal levels of serum potassium and magnesium are correlated with better OS in the multivariable analyses, suggesting possible mechanism(s) that may involve a biased postoperative diet, digestive tract malabsorption, or increased renal excretion among others in certain GC patients. ${ }^{25}$ Because ion metabolisms are important for life, the present findings warrant further investigations into ionic mechanisms that may be involved in cancer progression and prognosis of GC. On a different front, cell differentiation and HDL-C levels of more than $10.4 \mathrm{mmol} / \mathrm{L}$ are known predictors of $\mathrm{OS}^{26,27}$ in agreement with our observations reported here.

The nomogram analysis using bio-indicators to predict OS among GC patients has several clinical implications. First, these bio-indicators are clinical routine tests and easily obtainable in any clinical setting. Second, these bioindicators can be obtained prior to surgery giving clinicians room to elaborate treatment options in terms of quality of life and estimated length of survival for their patients. Third, the nomogram analysis provides a good $\mathrm{C}$-index, an ideal DCA, and a calibration curve in terms of predicting OS which is a reliable endpoint for retrospective studies. Fourth, different from other studies, ${ }^{7-10}$ our prediction algorithm provides a quantifiable risk score for individual patient counseling before surgery. Finally, it should be mentioned that the patients studied here were from Shihezi City, a city in China's far west, where a vast majority of residents are migrants from all over the country, suggesting heterogeneity in the nature of our patient population. ${ }^{11}$ This heterogeneity suggests that the nomogram algorithm developed may be useful in other geographical areas of China. These advantages suggest that the nomogram algorithm is equal to or better than the prediction algorithm of the TNM staging in terms of 5-year overall survival among GC patients after surgery.

The present study has several limitations. First, this is a single-center study whose applicability needs to be validated in the external center(s), including domestic as well as international centers with larger sample sizes, which will be our ongoing collaborative investigations. Second, due to differences in genetic and environmental backgrounds, the results and proposals from this study may have potential applicability in China and perhaps in Eastern countries, but apparently not so in Western countries before validation studies are performed. Third, ECOG PS (Eastern Cooperative Oncology Group performance status) score has not been included in this nomogram 
model due largely to its unstable predictive ability as demonstrated by a survey from the St. James's Hospital Cancer Center (Dublin, Ireland). ${ }^{28}$ Nevertheless, future validation studies considering the abovementioned factors would certainly advance the applicability of this nomogram model for predicting OS among GC patients.

\section{Conclusions}

We have developed a nomogram model based on the combination of several preoperatively obtained clinical bio-indicators and patients' basic features. The nomogram model allows the prediction of 5-year overall survival after radical gastrectomy for gastric cancer patients in a migrant city in the west of China. The novel nomogram provides significantly better clinical benefits than the 8th AJCC TNM staging system. The nomogram also provides an individualized prediction of survival, which may help clinicians to strategize treatment options prior to surgery and follow-up intervals after surgery.

\section{Abbreviations}

ECOG PS, Eastern Cooperative Oncology Group performance status; GC,gastric cancer; AGC, advanced gastric cancer; OS, overall survival; CI, confidence interval; AJCC, American Joint Committee on Cancer; ROC, receiver operating characteristic; AUC, area under curve; DCA, decision curve analysis; HR, hazard ratio; NPV, negative predictive value; PPV, positive predictive value; vs, versus; $\mathrm{T}$, tumor invasion; $\mathrm{N}$, lymph node metastasis; $\mathrm{M}$, distant metastasis; BMI, body mass index; UW, underweight; NW, normal weight; OW, overweight; OB, obesity; TP, total protein; Alb, albumin; $\mathrm{A} / \mathrm{G}$, albumin and globulin; Glu, blood glucose; TBIL, total bilirubin; DBIL, direct bilirubin; TC, total cholesterol; TG, triglyceride; HDL-C, high-density lipoprotein cholesterol; LDL-C, lowdensity lipoprotein cholesterol.

\section{Data sharing statement}

All the data generated or analyzed during this study are included in this article.

\section{Acknowledgments}

We sincerely thank all patients participated in this investigation. We are grateful to the Ministry of Science and Technology of China, the Bureau of Science and Technology of the Xinjiang Corps, and Shihezi University for their generous financial support.

\section{Author contributions}

All authors contributed to data analysis, drafting or revising the article, gave final approval of the version to be published, and agree to be accountable for all aspects of the work.

\section{Disclosure}

The authors report no conflicts of interest in this work.

\section{References}

1. Bray F, Ferlay J, Soerjomataram I, et al. Global cancer statistics 2018: GLOBOCAN estimates of incidence and mortality worldwide for 36 cancers in 185 countries. CA Cancer J Clin. 2018;68:394-424. doi: $10.3322 /$ caac. 21492

2. Chen W, Zheng R, Baade PD, et al. Cancer statistics in China, 2015. CA Cancer J Clin. 2016;66:115-132. doi:10.3322/caac.21338

3. Song KY, Park YG, Jeon HM, et al. A nomogram for predicting individual survival of patients with gastric cancer who underwent radical surgery with extended lymph node dissection. Gastric Cancer. 2014;17:287-293. doi:10.1007/s10120-013-0270-x

4. Amin MB, Edge S, Greene F, et al. AJCC Cancer Staging Manual [M]. 8th. New York: Springer; 2017. doi:10.1007/978-3-319-40618-3

5. Japanese Gastric Cancer Association. Japanese gastric cancer treatment guidelines 2014 (ver. 4). Gastric Cancer. 2017;20(1):1-19. doi:10.1007/s10120-016-0622-4

6. Thrumurthy SG, Chaudry MA, Hochhauser D, et al. The diagnosis and management of gastric cancer. BMJ. 2013;347:f6367-f6367. doi:10.1136/bmj.f6367

7. Kattan MW, Karpeh MS, Mazumdar M, et al. Postoperative nomogram for disease-specific survival after an R0 resection for gastric carcinoma. J Clin Oncol. 2003;21:3647-3650. doi:10.1200/ JCO.2003.01.240

8. Marrelli D, De Stefano A, de Manzoni G, et al. Prediction of recurrence after radical surgery for gastric cancer: a scoring system obtained from a prospective multicenter study. Ann Surg. 2005;241:247-255. doi:10.1097/01.sla.0000152019.14741.97

9. Han DS, Suh YS, Kong SH, et al. Nomogram predicting long-term survival after d2 gastrectomy for gastric cancer. J Clin Oncol. 2012;30:3834-3840. doi:10.1200/JCO.2012.41.8343

10. Hirabayashi S, Kosugi S, Isobe Y, et al. Development and external validation of a nomogram for overall survival after curative resection in serosa-negative, locally advanced gastric cancer. Ann Oncol. 2014;25:1179-1184. doi:10.1093/annonc/mdu125

11. Johnson C. Eurasian Corridors of Interconnection [M]. 2013. doi:10.4324/9780203796443

12. Zhou BF. Predictive values of body mass index and waist circumference for risk factors of certain related diseases in Chinese adults: study on optimal cut-off points of body mass index and waist circumference in Chinese adults. Asia Pac J of Clin Nutr. 2003;11 (Suppl 8):S685-693. doi:10.1046/j.1440-6047.11.s8.9.x

13. Shang H, Chen WX, Pan BS, et al. Reference intervals for common clinical biochemistry tests Part 2: serum total protein and albumin [M]. WS/T404.2-2012. Chinese. Available from: http://www.nhc.gov. $\mathrm{cn} /$ wjw/s9492/wsbz.shtml.

14. Shang H, Zhang J, Chen WX, et al. Reference intervals for common clinical biochemistry tests Part 3: serum potassium, sodium and chloride [M]. WS/T404.3-2012. Chinese. Available from: http:// www.nhc.gov.cn/wjw/s9492/wsbz.shtml.

15. Shang H, Pan BS, Chen WX, et al. Reference intervals for common clinical biochemistry tests Part 4: serum total bilirubin and direct bilirubin [M]. WS/T404.4-2018. Chinese. Available from: http:// www.nhc.gov.cn/wjw/s9492/wsbz.shtml. 
16. Shang H, Hao XK, Pan BS, et al. Reference intervals for common clinical biochemistry tests Part 6: serum calcium, phosphorus, magnesium, iron [M]. WS/T404.6-2015. Chinese. Available from: http:// www.nhc.gov.cn/wjw/s9492/wsbz.shtml.

17. Little RJA, Rubin DB. Statistical analysis with missing data. Technometrics. 2002;45(4):364-365. doi:10.1198/tech.2003.s167

18. Yu GZ, Chen Y, Wang JJ, et al. Overexpression of Grb2/HER2 signaling in Chinese gastric cancer: their relationship with clinicopathological parameters and prognostic significance. J Cancer Res Clin. 2009;135:1331-1339. doi:10.1007/s00432-009-0574-8

19. Vanek VW, Seballos RM, Chong D, et al. Serum potassium concentrations in trauma patients. South Med J. 1994;87:41-46. doi:10.1097/00007611-199401000-00010

20. Schlesinger-Raab A, Mihaljevic AJ, Egert S, et al. Outcome of gastric cancer in the elderly: a population-based evaluation of the Munich Cancer Registry. Gastric Cancer. 2016;19:713-722. doi:10.1007/s10120-015-0527-7

21. Chen HN, Chen XZ, Zhang WH, et al. The impact of body mass index on the surgical outcomes of patients with gastric cancer: a 10-year, single-institution cohort study. Medicine. 2015;94:e1769. doi:10.1097/md.0000000000001769

22. Liu BZ, Tao L, Chen YZ, et al. Preoperative body mass index, blood albumin and triglycerides predict survival for patients with gastric cancer. PLoS One. 2016;11:e0157401. doi:10.1371/journal.pone.0157401
23. Lipkin M, Newmark H. Effect of added dietary calcium on colonic epithelial-cell proliferation in subjects at high risk for familial colonic cancer. New Engl J Med. 1985;313:1381-1384. doi:10.1056/ NEJM198511283132203

24. Lin Q. The lever of serum vitamin D and calcium iron and the clinic significance in patients with gastric cancer [D]. Jinan: Shandong University; 2010. Chinese. doi: 10.7666/d.y1792812

25. Raghunand N, He X, Van Sluis R, et al. Enhancement of chemotherapy by manipulation of tumor pH. Brit J Cancer. 1999;80 (7):1005-1011. doi:10.1038/sj.bjc.6690455

26. Lipham JC, DeMeester TR. Preoperative diagnosis of lymph node metastasis: a dream? Gastric Cancer. 2006;9:70-71. doi:10.1007/ s10120-006-0382-7

27. Kitahara CM, BdG A, Freedman ND, et al. Total cholesterol and cancer risk in a large prospective study in Korea. J Clin Oncol. 2011;29:1592-1598. doi:10.1200/JCO.2010.31.5200

28. Broderick JM, Hussey J, Kennedy MJ, et al. Patients over 65 years are assigned lower ECOG PS scores than younger patients, although objectively measured physical activity is no different. J Geriatr Oncol. 2014;5(1):49-56. doi:10.1016/j.jgo.2013.07.010

\section{Publish your work in this journal}

Cancer Management and Research is an international, peer-reviewed open access journal focusing on cancer research and the optimal use of preventative and integrated treatment interventions to achieve improved outcomes, enhanced survival and quality of life for the cancer patient.
The manuscript management system is completely online and includes a very quick and fair peer-review system, which is all easy to use. Visit http://www.dovepress.com/testimonials.php to read real quotes from published authors. 